\title{
Upper and Lower Solutions Method for a Class of Singular Fractional Boundary Value Problems with $p$-Laplacian Operator
}

\author{
Jinhua Wang and Hongjun Xiang \\ Department of Mathematics, Xiangnan University, Chenzhou 423000, China \\ Correspondence should be addressed to Hongjun Xiang, hunxhjxhj67@126.com
}

Received 24 May 2010; Accepted 25 August 2010

Academic Editor: Paul Eloe

Copyright (c) $2010 \mathrm{~J}$. Wang and H. Xiang. This is an open access article distributed under the Creative Commons Attribution License, which permits unrestricted use, distribution, and reproduction in any medium, provided the original work is properly cited.

The upper and lower solutions method is used to study the $p$-Laplacian fractional boundary value problem $D_{0^{+}}^{\gamma}\left(\phi_{p}\left(D_{0+}^{\alpha} u(t)\right)\right)=f(t, u(t)), 0<t<1, u(0)=0, u(1)=a u(\xi), D_{0+}^{\alpha} u(0)=0$, and $D_{0+}^{\alpha} u(1)=b D_{0+}^{\alpha} u(\eta)$, where $1<\alpha, \gamma \leqslant 2,0 \leqslant a, b \leqslant 1,0<\xi, \eta<1$. Some new results on the existence of at least one positive solution are obtained. It is valuable to point out that the nonlinearity $f$ can be singular at $t=0,1$ or $u=0$.

\section{Introduction}

It is well know that the upper and lower solutions method is a powerful tool for proving the existence results for boundary value problem. It has been used to deal with many multipoint boundary value problem of integer ordinary differential equations (see, e.g., [1-3] and references therein).

Recently, boundary value problems of nonlinear fractional differential equations have aroused considerable attention. Many people pay attention to the existence and multiplicity of solutions or positive solutions for boundary value problems of nonlinear fractional differential equations by means of some fixed point theorems, such as the Krasnosel'skii fixed-point theorem, the Leggett-Williams fixed-point theorem, and the Schauder fixed-point theorem (see [4-8]). To the best of our knowledge, the upper and lower solutions method is seldom considered in the literatures, and there are few papers devoted to investigate $p$ Laplacian fractional boundary value problems.

In this paper, we deal with the following $p$-Laplacian fractional boundary value problem:

$$
\begin{gathered}
D_{0+}^{\gamma}\left(\phi_{p}\left(D_{0+}^{\alpha} u(t)\right)\right)=f(t, u(t)), \quad 0<t<1, \\
u(0)=0, \quad u(1)=a u(\xi), \quad D_{0+}^{\alpha} u(0)=0, \quad D_{0+}^{\alpha} u(1)=b D_{0+}^{\alpha} u(\eta),
\end{gathered}
$$


where $1<\alpha, \gamma \leqslant 2,0 \leqslant a, b \leqslant 1,0<\xi, \eta<1$, and $D^{\alpha}$ is the standard Riemann-Liouville fractional differential operator of order $\alpha, \phi_{p}(s)=|s|^{p-2} s, p>1,\left(\phi_{p}\right)^{-1}=\phi_{q},(1 / p)+(1 / q)=$ 1. By using upper and lower solutions method, the existence results of at least one positive solution for the above fractional boundary value problem are established, and an example is given to show the effectiveness of our results. It is valuable to point out that the nonlinearity $f$ can be singular at $t=0,1$ or $u=0$.

The remaining part of the paper is organized as follows. In Section 2, we will present some definitions and lemmas. In Section 3, some results are given. In Section 4, we present an example to demonstrate our results.

\section{Basic Definitions and Preliminaries}

In this section, we present some necessary definitions and lemmas.

Definition 2.1 (see [9]). The integral

$$
I_{0+}^{\alpha} y(t)=\frac{1}{\Gamma(\alpha)} \int_{0}^{t}(t-s)^{\alpha-1} y(s) d s
$$

where $\alpha>0$, is called the Riemann-Liouville fractional integral of order $\alpha$.

Definition 2.2 (see [10]). For a function $y(t)$ given in the interval $[0, \infty)$, the expression

$$
D_{0+}^{\alpha} y(t)=\frac{1}{\Gamma(n-\alpha)}\left(\frac{d}{d t}\right)^{n} \int_{0}^{t}(t-s)^{n-\alpha-1} y(s) d s
$$

where $n=[\alpha]+1$, and $[\alpha]$ denotes the integer part of number $\alpha$, is called the RiemannLiouville fractional derivative of order $\alpha$.

Remark 2.3. From the definition of the Riemann-Liouville fractional derivative, we quote for $\mu>-1$, then

$$
D_{0+}^{\alpha} t^{\mu}=\frac{\Gamma(1+\mu)}{\Gamma(1+\mu-\alpha)} t^{\mu-\alpha}
$$

In particular, $D_{0+}^{\alpha} t^{\alpha-m}=0, m=1,2, \ldots, N$, where $N$ is the smallest integer greater than or equal to $\alpha$.

Lemma 2.4 (see [4]). Assume that $u \in C(0,1) \cap L(0,1)$ with a fractional derivative of order $\alpha>0$ that belongs to $C(0,1) \cap L(0,1)$. Then

$$
I_{0+}^{\alpha} D_{0+}^{\alpha} u(t)=u(t)+C_{1} t^{\alpha-1}+C_{2} t^{\alpha-2}+\cdots+C_{N} t^{\alpha-N},
$$

for some $C_{i} \in R, i=1,2, \ldots N$, where $N$ is the smallest integer greater than or equal to $\alpha$. 
Lemma 2.5 (see [6]). Let $y \in C[0,1]$ and $1<\alpha \leqslant 2$, the unique solution of

$$
\begin{gathered}
D_{0+}^{\alpha} u(t)+y(t)=0, \quad 0<t<1, \\
u(0)=0, \quad u(1)=a u(\xi),
\end{gathered}
$$

is

$$
u(t)=\int_{0}^{1} G(t, s) y(s) d s,
$$

where

$$
G(t, s)= \begin{cases}\frac{[t(1-s)]^{\alpha-1}-a t^{\alpha-1}(\xi-s)^{\alpha-1}-(t-s)^{\alpha-1}\left(1-a \xi^{\alpha-1}\right)}{\left(1-a \xi^{\alpha-1}\right) \Gamma(\alpha)}, & 0 \leqslant s \leqslant t \leqslant 1, s \leqslant \xi, \\ \frac{[t(1-s)]^{\alpha-1}-(t-s)^{\alpha-1}\left(1-a \xi^{\alpha-1}\right)}{\left(1-a \xi^{\alpha-1}\right) \Gamma(\alpha)}, & 0<\xi \leqslant s \leqslant t \leqslant 1, \\ \frac{[t(1-s)]^{\alpha-1}-a t^{\alpha-1}(\xi-s)^{\alpha-1}}{\left(1-a \xi^{\alpha-1}\right) \Gamma(\alpha)}, & 0 \leqslant t \leqslant s \leqslant \xi \leqslant 1, \\ \frac{[t(1-s)]^{\alpha-1}}{\left(1-a \xi^{\alpha-1}\right) \Gamma(\alpha)}, & 0 \leqslant t \leqslant s \leqslant 1, \xi \leqslant s .\end{cases}
$$

Lemma 2.6. Let $y \in C[0,1], 1<\alpha, \gamma \leqslant 2,0<\xi, \eta<1,0 \leqslant a$, and $b \leqslant 1$. The fractional boundary value problem

$$
\begin{gathered}
D_{0+}^{\gamma}\left(\phi_{p}\left(D_{0+}^{\alpha} u(t)\right)\right)=y(t), \quad 0<t<1, \\
u(0)=0, \quad u(1)=a u(\xi), \quad D_{0+}^{\alpha} u(0)=0, \quad D_{0+}^{\alpha} u(1)=b D_{0+}^{\alpha} u(\eta),
\end{gathered}
$$

has a unique solution

$$
u(t)=\int_{0}^{1} G(t, s) \phi_{q}\left(\int_{0}^{1} H(s, \tau) y(\tau) d \tau\right) d s
$$


where

$$
H(t, s)= \begin{cases}\frac{[t(1-s)]^{\gamma-1}-b_{1} t^{\gamma-1}(\eta-s)^{\gamma-1}-(t-s)^{\gamma-1}\left(1-b_{1} \eta^{\gamma-1}\right)}{\left(1-b_{1} \eta^{\gamma-1}\right) \Gamma(\gamma)}, & 0 \leqslant s \leqslant t \leqslant 1, s \leqslant \eta, \\ \frac{[t(1-s)]^{\gamma-1}-(t-s)^{\gamma-1}\left(1-b_{1} \eta^{\gamma-1}\right)}{\left(1-b_{1} \eta^{\gamma-1}\right) \Gamma(\gamma)}, & 0<\eta \leqslant s \leqslant t \leqslant 1, \\ \frac{[t(1-s)]^{\gamma-1}-b_{1} t^{\gamma-1}(\eta-s)^{\gamma-1}}{\left(1-b_{1} \eta^{\gamma-1}\right) \Gamma(\gamma)}, & 0 \leqslant t \leqslant s \leqslant \eta \leqslant 1, \\ \frac{[t(1-s)]^{\gamma-1}}{\left(1-b_{1} \eta^{\gamma-1}\right) \Gamma(\gamma)}, & 0 \leqslant t \leqslant s \leqslant 1, \eta \leqslant s,\end{cases}
$$

$b_{1}=b^{p-1}$ and $G(t, s)$ is defined by (2.7).

Proof. At first, by Lemma 2.4, the (2.8) is equivalent to the integral equation

$$
\phi_{p}\left(D_{0+}^{\alpha} u(t)\right)=I_{0+}^{\gamma} y(t)+C_{1} t^{\gamma-1}+C_{2} t^{\gamma-2}, \quad C_{1}, C_{1} \in R,
$$

that is,

$$
\phi_{p}\left(D_{0+}^{\alpha} u(t)\right)=\int_{0}^{t} \frac{(t-\tau)^{\gamma-1}}{\Gamma(\gamma)} y(\tau) d \tau+C_{1} t^{\gamma-1}+C_{2} t^{\gamma-2} .
$$

By the boundary conditions $D_{0+}^{\alpha} u(0)=0, D_{0+}^{\alpha} u(1)=b D_{0+}^{\alpha} u(\eta)$, we have

$$
C_{2}=0, \quad C_{1}=-\int_{0}^{1} \frac{(1-\tau)^{\gamma-1}}{\Gamma(\gamma)\left(1-b_{1} \eta^{\gamma-1}\right)} y(\tau) d \tau+\int_{0}^{\eta} \frac{b_{1}(\eta-\tau)^{\gamma-1}}{\Gamma(\gamma)\left(1-b_{1} \eta^{\gamma-1}\right)} y(\tau) d \tau
$$

Therefore, the solution $u(t)$ of boundary value problem (2.8) satisfies

$$
\begin{aligned}
\phi_{p}\left(D_{0+}^{\alpha} u(t)\right)= & \int_{0}^{t} \frac{(t-\tau)^{\gamma-1}}{\Gamma(\gamma)} y(\tau) d \tau-\int_{0}^{1} \frac{t^{\gamma-1}(1-\tau)^{\gamma-1}}{\Gamma(\gamma)\left(1-b_{1} \eta^{\gamma-1}\right)} y(\tau) d \tau \\
& +\int_{0}^{\eta} \frac{b_{1} t^{\gamma-1}(\eta-\tau)^{\gamma-1}}{\Gamma(\gamma)\left(1-b_{1} \eta^{\gamma-1}\right)} y(\tau) d \tau \\
= & -\int_{0}^{1} H(t, \tau) y(\tau) d \tau .
\end{aligned}
$$


Consequently, $D_{0+}^{\alpha} u(t)+\phi_{q}\left(\int_{0}^{1} H(t, \tau) y(\tau) d \tau\right)=0$. So, fractional boundary value problem (2.8) is equivalent to the following problem:

$$
\begin{gathered}
D_{0+}^{\alpha} u(t)+\phi_{q}\left(\int_{0}^{1} H(t, \tau) y(\tau) d \tau\right)=0, \quad 0<t<1, \\
u(0)=0, \quad u(1)=a u(\xi) .
\end{gathered}
$$

Lemma 2.5 implies that fractional boundary value problem (2.8) has a unique solution

$$
u(t)=\int_{0}^{1} G(\mathrm{t}, s) \phi_{q}\left(\int_{0}^{1} H(s, \tau) y(\tau) d \tau\right) d s
$$

The proof is completed.

Lemma 2.7. Let $0 \leqslant a, b \leqslant 1,0<\xi, \eta<1,1<\alpha$, and $\gamma \leqslant 2$. The functions $G(t, s)$ and $H(t, s)$ defined by (2.7) and (2.10), respectively, are continuous on $[0,1] \times[0,1]$ and satisfy

(1) $G(t, s) \geqslant 0, H(t, s) \geqslant 0$, for $t, s \in[0,1]$;

(2) $G(t, s) \leqslant G(s, s), H(t, s) \leqslant H(s, s)$ for $t, s \in[0,1]$;

(3) $G(t, s) / G(1, s) \geqslant t^{\alpha-1}$, for $t, s \in(0,1)$.

Proof. The proofs of part (1) and part (2) can be obtained in [6]. Here, we only prove part (3). If $0<s \leqslant t<1, s \leqslant \xi$, then

$$
\begin{aligned}
\frac{G(t, s)}{G(1, s)} & =\frac{[t(1-s)]^{\alpha-1}-a t^{\alpha-1}(\xi-s)^{\alpha-1}-(t-s)^{\alpha-1}\left(1-a \xi^{\alpha-1}\right)}{a \xi^{\alpha-1}(1-s)^{\alpha-1}-a(\xi-s)^{\alpha-1}} \\
& =t^{\alpha-1} \cdot \frac{(1-s)^{\alpha-1}-a(\xi-s)^{\alpha-1}-(1-(s / t))^{\alpha-1}\left(1-a \xi^{\alpha-1}\right)}{a \xi^{\alpha-1}(1-s)^{\alpha-1}-a(\xi-s)^{\alpha-1}} \geqslant t^{\alpha-1} .
\end{aligned}
$$

If $0<\xi \leqslant s \leqslant t<1$, then

$$
\begin{aligned}
\frac{G(t, s)}{G(1, s)} & =\frac{[t(1-s)]^{\alpha-1}-(t-s)^{\alpha-1}\left(1-a \xi^{\alpha-1}\right)}{a \xi^{\alpha-1}(1-s)^{\alpha-1}} \\
& =t^{\alpha-1} \cdot \frac{(1-s)^{\alpha-1}-(1-(s / t))^{\alpha-1}\left(1-a \xi^{\alpha-1}\right)}{a \xi^{\alpha-1}(1-s)^{\alpha-1}} \geqslant t^{\alpha-1}
\end{aligned}
$$

If $0<t \leqslant s \leqslant \xi<1$, then

$$
\begin{aligned}
\frac{G(t, s)}{G(1, s)} & =\frac{[t(1-s)]^{\alpha-1}-a t^{\alpha-1}(\xi-s)^{\alpha-1}}{a \xi^{\alpha-1}(1-s)^{\alpha-1}-a(\xi-s)^{\alpha-1}} \\
& =t^{\alpha-1} \cdot \frac{(1-s)^{\alpha-1}-a(\xi-s)^{\alpha-1}}{a \xi^{\alpha-1}(1-s)^{\alpha-1}-a(\xi-s)^{\alpha-1}} \geqslant t^{\alpha-1} .
\end{aligned}
$$


If $0<t \leqslant s<1, \xi \leqslant s$, then

$$
\frac{G(t, s)}{G(1, s)}=\frac{[t(1-s)]^{\alpha-1}}{a \xi^{\alpha-1}(1-s)^{\alpha-1}}=t^{\alpha-1} \cdot \frac{(1-s)^{\alpha-1}}{a \xi^{\alpha-1}(1-s)^{\alpha-1}} \geqslant t^{\alpha-1} .
$$

The above inequalities imply that part (3) of Lemma 2.7 holds.

From Lemmas 2.5 and 2.7, it is easy to obtain the following lemma.

Lemma 2.8. Let $0 \leqslant a \leqslant 1,0<\xi<1,1<\alpha \leqslant 2$. If $y(t) \in C[0,1]$, and $y(t) \geqslant 0$, then fractional boundary value problem (2.5) has a unique solution $u(t) \geqslant 0, t \in[0,1]$.

Let $E=\left\{u: u, \phi_{p}\left(D_{0+}^{\alpha} u\right) \in C^{2}[0,1]\right\}$. Now one introduces the following definitions about the upper and lower solutions of fractional boundary value problem (1.1).

Definition 2.9. A function $\alpha(t)$ is called a lower solution of fractional boundary value problem (1.1), if $\alpha(t) \in E$ and $\alpha(t)$ satisfies

$$
\begin{gathered}
D_{0+}^{\gamma}\left(\phi_{p}\left(D_{0+}^{\alpha} \alpha(t)\right)\right) \leqslant f(t, \alpha(t)), \quad 0<t<1,1<\alpha, \gamma \leqslant 2, \\
\alpha(0) \leqslant 0, \alpha(1) \leqslant a \alpha(\xi) \\
D_{0+}^{\alpha} \alpha(0) \geqslant 0, D_{0+}^{\alpha} \alpha(1) \geqslant b D_{0+}^{\alpha} \alpha(\eta) .
\end{gathered}
$$

Definition 2.10. A function $\beta(t)$ is called an upper solution of fractional boundary value problem (1.1), if $\beta(t) \in E$ and $\beta(t)$ satisfies

$$
\begin{gathered}
D_{0+}^{\gamma}\left(\phi_{p}\left(D_{0+}^{\alpha} \beta(t)\right)\right) \geqslant f(t, \beta(t)), \quad 0<t<1,1<\alpha, \gamma \leqslant 2, \\
\beta(0) \geqslant 0, \quad \beta(1) \geqslant a \beta(\xi), \\
D_{0+}^{\alpha} \beta(0) \leqslant 0, \quad D_{0+}^{\alpha} \beta(1) \leqslant b D_{0+}^{\alpha} \beta(\eta) .
\end{gathered}
$$

\section{Main Result}

In this section, our objective is to give and prove our main results.

For the sake of simplicity, we assume that

$\left(H_{1}\right) f(t, u) \in C[(0,1) \times(0,+\infty),[0,+\infty)]$ and $f(t, u)$ is nonincreasing relative to $u$;

$\left(H_{2}\right)$ For any constant $\mu>0,0<\int_{0}^{1} H(s, s) f\left(s, \mu s^{\alpha-1}\right) d s<+\infty$;

$\left(H_{3}\right)$ There exist a continuous function $m(t)$ and a positive number $\lambda$ such that $m(t) \geqslant$ $\lambda t^{\alpha-1}, t \in[0,1]$, and

$$
\begin{gathered}
\int_{0}^{1} G(t, s) \phi_{q}\left(\int_{0}^{1} H(s, \tau) f(\tau, m(\tau)) d \tau\right) d s=n(t) \geqslant m(t), \\
\int_{0}^{1} G(t, s) \phi_{q}\left(\int_{0}^{1} H(s, \tau) f(\tau, n(\tau)) d \tau\right) d s \geqslant m(t) .
\end{gathered}
$$


We define $P=:\left\{u \in C[0,1]\right.$ : there exists a number $\lambda_{u}>0$ such that $u(t) \geqslant \lambda_{u} t^{\alpha-1}, t \in$ $[0,1]\}$. And define an operator $T$ by

$$
T u(t)=\int_{0}^{1} G(t, s) \phi_{q}\left(\int_{0}^{1} H(s, \tau) f(\tau, u(\tau)) d \tau\right) d s, \quad \forall u \in P .
$$

Theorem 3.1. Suppose that conditions $\left(H_{1}\right) \sim\left(H_{3}\right)$ are satisfied, then the boundary value problem (1.1) has at least one positive solution $\rho(t)$, which satisfies $\rho(t) \geqslant \mu t^{\alpha-1}$ for some positive number $\mu$.

Proof. We will divide our proof into four steps.

Step 1 . We prove that $T(P) \subseteq P$.

Firstly, from Lemma 2.7 and conditions $\left(H_{1}\right) \sim\left(H_{2}\right)$, for any $u \in P$, there exists $\lambda_{u}>0$, such that

$$
\int_{0}^{1} H(s, \tau) f(\tau, u(\tau)) d \tau \leqslant \int_{0}^{1} H(\tau, \tau) f\left(\tau, \lambda_{u} \tau^{\alpha-1}\right) d \tau<+\infty
$$

Thus,

$$
\begin{aligned}
T u(t) & =\int_{0}^{1} G(t, s) \phi_{q}\left(\int_{0}^{1} H(s, \tau) f(\tau, u(\tau)) d \tau\right) d s \\
& \leqslant \int_{0}^{1} G(s, s) d s \cdot \phi_{q}\left(\int_{0}^{1} H(\tau, \tau) f\left(\tau, \lambda_{u} \tau^{\alpha-1}\right) d \tau\right) \\
& <+\infty
\end{aligned}
$$

Secondly, it follows from Lemma 2.7 that

$$
\begin{aligned}
T u(t) & =\int_{0}^{1} \frac{G(t, s)}{G(1, s)} G(1, s) \phi_{q}\left(\int_{0}^{1} H(s, \tau) f(\tau, u(\tau)) d \tau\right) d s \\
& \geqslant t^{\alpha-1} \int_{0}^{1} G(1, s) \phi_{q}\left(\int_{0}^{1} H(s, \tau) f(\tau, u(\tau)) d \tau\right) d s \\
& =\lambda_{T u} t^{\alpha-1}, \quad \forall t \in[0,1] .
\end{aligned}
$$

Consequently, $T$ is well defined and $T(P) \subseteq P$.

In the meanwhile, by direct computations, we can obtain

$$
\begin{gathered}
D_{0+}^{\gamma}\left(\phi_{p}\left(D_{0+}^{\alpha}(T u)(t)\right)\right)=f(t, u(t)), \quad 0<t<1,1<\alpha, \gamma \leqslant 2 \\
(T u)(0)=0, \quad(T u)(1)=a(T u)(\xi) \\
D_{0+}^{\alpha}(T u)(0)=0, \quad D_{0+}^{\alpha}(T u)(1)=b D_{0+}^{\alpha}(T u)(\eta)
\end{gathered}
$$


Step 2. We will prove that the functions $\alpha(t)=\operatorname{Tn}(t), \beta(t)=\operatorname{Tm}(t)$ are lower and upper solutions of fractional boundary value problem (1.1), respectively.

From $\left(H_{1}\right)$, we know that $T$ is nonincreasing relative to $u$. Combining $\left(H_{3}\right)$, we have

$$
m(t) \leqslant n(t)=\operatorname{Tm}(t), \quad \operatorname{Tn}(t) \leqslant n(t)=\operatorname{Tm}(t), \quad t \in[0,1] .
$$

Therefore, $\alpha(t) \leqslant \beta(t)$.

By Step 1, we know $\alpha(t), \beta(t) \in P$. And by (3.6) (3.8), we obtain

$$
\begin{gathered}
D_{0+}^{\gamma}\left(\phi_{p}\left(D_{0+}^{\alpha}(\alpha)(t)\right)\right)-f(t, \alpha(t)) \leqslant D_{0+}^{\gamma}\left(\phi_{p}\left(D_{0+}^{\alpha}(T n)(t)\right)\right)-f(t, n(t))=0, \\
\alpha(0)=0, \quad \alpha(1)=a \alpha(\xi), \quad D_{0+}^{\alpha} \alpha(0)=0, \quad D_{0+}^{\alpha} \alpha(1)=b D_{0+}^{\alpha} \alpha(\eta), \\
D_{0+}^{\gamma}\left(\phi_{p}\left(D_{0+}^{\alpha}(\beta)(t)\right)\right)-f(t, \beta(t)) \geqslant D_{0+}^{\gamma}\left(\phi_{p}\left(D_{0+}^{\alpha}(T m)(t)\right)\right)-f(t, m(t))=0, \\
\beta(0)=0, \quad \beta(1)=a \beta(\xi), \quad D_{0+}^{\alpha} \beta(0)=0, \quad D_{0+}^{\alpha} \beta(1)=b D_{0+}^{\alpha} \beta(\eta),
\end{gathered}
$$

that is, $\alpha(t)$ and $\beta(t)$ are lower and upper solutions of fractional boundary value problem (1.1), respectively.

Step 3. We will show that the fractional boundary value problem

$$
\begin{gathered}
D_{0+}^{\gamma}\left(\phi_{p}\left(D_{0+}^{\alpha} u(t)\right)\right)=g(t, u(t)), \quad 0<t<1,1<\alpha, \gamma \leqslant 2, \\
u(0)=0, \quad u(1)=a u(\xi), \quad D_{0+}^{\alpha} u(0)=0, \quad D_{0+}^{\alpha} u(1)=b D_{0+}^{\alpha} u(\eta),
\end{gathered}
$$

has a positive solution, where

$$
g(t, u(t))= \begin{cases}f(t, \alpha(t)) & \text { if } u(t)<\alpha(t) \\ f(t, u(t)) & \text { if } \alpha(t) \leqslant u(t) \leqslant \beta(t) \\ f(t, \beta(t)) & \text { if } u(t)>\beta(t)\end{cases}
$$

Thus, we consider the operator $A: C[0,1] \rightarrow C[0,1]$ defined as follows:

$$
A u(t)=\int_{0}^{1} G(t, s) \phi_{q}\left(\int_{0}^{1} H(s, \tau) g(\tau, u(\tau)) d \tau\right) d s
$$

where $G(t, s)$ is defined as $(2.7), H(t, s)$ is defined as (2.10). It is clear that $A u \geqslant 0$, for all $u \in$ $P$, and a fixed point of the operator $A$ is a solution of the fractional boundary value problem (3.10). 
Since $\alpha(t) \in P$, there exists a positive number $\lambda_{\alpha}$ such that $\alpha(t) \geqslant \lambda_{\alpha} t^{\alpha-1}, t \in[0,1]$. It follows from $\left(H_{2}\right)$ that

$$
\begin{aligned}
\int_{0}^{1} H(\tau, \tau) g(\tau, \alpha(\tau)) d \tau & \leqslant \int_{0}^{1} H(\tau, \tau) f(\tau, \alpha(\tau)) d \tau \\
& \leqslant \int_{0}^{1} H(\tau, \tau) f\left(\tau, \lambda_{\alpha} \tau^{\alpha-1}\right) d \tau<+\infty
\end{aligned}
$$

Consequently, for all $u(t) \in C[0,1]$, we have

$$
\begin{aligned}
A u(t) & =\int_{0}^{1} G(t, s) \phi_{q}\left(\int_{0}^{1} H(s, \tau) g(\tau, u(\tau)) d \tau\right) d s \\
& \leqslant \int_{0}^{1} G(s, s) \phi_{q}\left(\int_{0}^{1} H(\tau, \tau) g(\tau, u(\tau)) d \tau\right) d s \\
& \leqslant \int_{0}^{1} G(s, s) d s \cdot \phi_{q}\left(\int_{0}^{1} H(\tau, \tau) f(\tau, \alpha(\tau)) d \tau\right)<+\infty,
\end{aligned}
$$

which implies that the operator $A$ is uniformly bounded.

On the other hand, since $G(t, s)$ is continuous on $[0,1] \times[0,1]$, it is uniformly continuous on $[0,1] \times[0,1]$. So, for fixed $s \in[0,1]$ and for any $\varepsilon>0$, there exists a constant $\delta>0$, such that any $t_{1}, t_{2} \in[0,1]$ and $\left|t_{1}-t_{2}\right|<\delta$,

$$
\left|G\left(t_{1}, s\right)-G\left(t_{2}, s\right)\right|<\frac{\varepsilon}{\phi\left(\int_{0}^{1}(\tau, \tau) f\left(\tau, \lambda_{\alpha} \tau^{\alpha-1}\right) d \tau\right)}
$$

Then, for all $u(t) \in C[0,1]$,

$$
\begin{aligned}
\left|\mathrm{A} u\left(t_{2}\right)-A u\left(t_{1}\right)\right| & \leqslant \int_{0}^{1}\left|G\left(t_{2}, s\right)-G\left(t_{1}, s\right)\right| \phi_{q}\left(\int_{0}^{1} H(\tau, \tau) g(\tau, u(\tau)) d \tau\right) d s \\
& \leqslant \int_{0}^{1}\left|G\left(t_{2}, s\right)-G\left(t_{1}, s\right)\right| \phi_{q}\left(\int_{0}^{1} H(\tau, \tau) f(\tau, \alpha(\tau)) d \tau\right) d s \\
& \leqslant \int_{0}^{1}\left|G\left(t_{2}, s\right)-G\left(t_{1}, s\right)\right| d s \cdot \phi_{q}\left(\int_{0}^{1} H(\tau, \tau) f\left(\tau, \lambda_{\alpha} \tau^{\alpha-1}\right) d \tau\right) \\
& <\varepsilon
\end{aligned}
$$

that is to say, $A$ is equicontinuous. Thus, from the Arzela-Ascoli Theorem, we know that $A$ is a compact operator. By the Schauder's fixed-point theorem, the operator $A$ has a fixed point; that is, the fractional boundary value problem (3.10) has a positive solution. 
Step 4. We will prove that fractional boundary value problem (1.1) has at least one positive solution.

Suppose that $\rho(t)$ is a solution of (3.10), we only need to prove that $\alpha(t) \leqslant \rho(t) \leqslant$ $\beta(t), t \in[0,1]$.

Let $\rho(t)$ be a solution of (3.10). We have

$$
\rho(0)=0, \quad \rho(1)=a \rho(\xi), \quad D_{0+}^{\alpha} \rho(0)=0, \quad D_{0+}^{\alpha} \rho(1)=b D_{0+}^{\alpha} \rho(\eta) .
$$

In addition, the function $f(t, u)$ is nonincreasing in $u$, we know that

$$
f(t, \beta(t)) \leqslant g(t, \rho(t)) \leqslant f(t, \alpha(t)), \quad t \in[0,1]
$$

It follows from (3.8) and $\left(H_{3}\right)$ that

$$
f(t, n(t)) \leqslant g(t, \rho(t)) \leq f(t, m(t)), \quad t \in[0,1]
$$

By (3.6) and $m(t) \in P$, we obtain

$$
D_{0+}^{\gamma}\left(\phi_{p}\left(D_{0+}^{\alpha} \beta(t)\right)\right)=D_{0+}^{\gamma}\left(\phi_{p}\left(D_{0+}^{\alpha}(\operatorname{Tm})(t)\right)\right)=f(t, m(t)), \quad t \in[0,1] .
$$

Together with (3.7), (3.17)-(3.20), we obtain

$$
\begin{gathered}
D_{0+}^{\gamma}\left(\phi_{p}\left(D_{0+}^{\alpha} \beta(t)\right)\right)-D_{0+}^{\gamma}\left(\phi_{p}\left(D_{0+}^{\alpha} \rho(t)\right)\right)=f(t, m(t))-g(t, \rho(t)) \geqslant 0, \quad t \in[0,1] \\
(\beta-\rho)(0)=0, \quad(\beta-\rho)(1)=a(\beta-\rho)(\xi) \\
D_{0+}^{\alpha}(\beta-\rho)(0)=0, \quad D_{0+}^{\alpha}(\beta-\rho)(1)=b D_{0+}^{\alpha}(\beta-\rho)(\eta) .
\end{gathered}
$$

Let $z(t)=\phi_{p}\left(D_{0+}^{\alpha} \beta(t)\right)-\phi_{p}\left(D_{0+}^{\alpha} \rho(t)\right)$, we obtain

$$
D_{0+}^{\gamma}\left(\phi_{p}\left(D_{0+}^{\alpha} \beta(t)\right)\right)-D_{0+}^{\gamma}\left(\phi_{p}\left(D_{0+}^{\alpha} \rho(t)\right)\right) \geqslant f(t, \beta(t))-g(t, \rho(t)) \geqslant 0, \quad t \in[0,1]
$$

and $z(0)=0, z(1)-\phi_{p}(b) z(\eta)=0$.

By Lemma 2.8, we know $z(t) \leqslant 0, t \in[0,1]$, which implies that

$$
\phi_{p}\left(D_{0+}^{\alpha} \beta(t)\right) \leqslant \phi_{p}\left(D_{0+}^{\alpha} \rho(t)\right), \quad t \in[0,1] .
$$

Since $\phi_{p}$ is monotone increasing, we have $D_{0+}^{\alpha} \beta(t) \leqslant D_{0+}^{\alpha} \rho(t)$, that is, $D_{0+}^{\alpha}(\beta-\rho)(t) \leqslant 0$. By Lemma 2.8 and (3.21), we have $(\beta-\rho)(t) \geqslant 0$. Therefore, $\beta(t) \geqslant \rho(t), t \in[0,1]$. 
In the similar way, we can prove that $\alpha(t) \leqslant \rho(t), t \in[0,1]$. Consequently, $\rho(t)$ is a positive solution of fractional boundary value problem (1.1). This completes the proof.

Theorem 3.2. If $f(t, u) \in C([0,1] \times[0,+\infty),[0,+\infty))$ is nonincreasing relative to $u$ and $f(t, u)$ does not vanish identically for any $u>0$, then the fractional boundary value problem (1.1) has at least one positive solution $\rho(t)$, which satisfies $\rho(t) \geqslant \mu t^{\alpha-1}$ for some positive number $\mu$.

The proof is similar to Theorem 3.1, we omit it here.

\section{Example}

Example 4.1. As an example, we consider the fractional boundary value problem

$$
\begin{gathered}
D_{0+}^{4 / 3}\left(\phi_{p}\left(D_{0+}^{3 / 2} u(t)\right)\right)=t^{2}+\frac{1}{\sqrt{u}}, \quad 0<t<1, \\
u(0)=0, \quad u(1)=\frac{1}{3} u\left(\frac{1}{2}\right), \quad D_{0+}^{3 / 2} u(0)=0, \quad D_{0+}^{3 / 2} u(1)=\frac{1}{2} D_{0+}^{3 / 2} u\left(\frac{2}{3}\right) .
\end{gathered}
$$

Proof. It is clear that $\left(H_{1}\right)$ holds. For any $\lambda>0$,

$$
\int_{0}^{1} H(\tau, \tau) f\left(\tau, \lambda \tau^{((3 / 2)-1)}\right) d \tau=\int_{0}^{1} H(\tau, \tau)\left(\tau^{2}+\frac{1}{\sqrt{\lambda} \tau^{1 / 4}}\right) d \tau<+\infty
$$

which implies that $\left(\mathrm{H}_{2}\right)$ holds.

For $0<r<1, f(t, u)=t^{2}+(1 / \sqrt{ } u) \leqslant t^{2}+(1 / \sqrt{ } r u) \leqslant r^{-1 / 2} f(t, u)$. Let $a(t)=t^{1 / 2}$, by (3.5), we have

$$
b(t)=: T a(t)=\int_{0}^{1} G(t, s) \phi_{q}\left(\int_{0}^{1} H(s, \tau) f(\tau, a(\tau)) d \tau\right) d s \in P,
$$

and $T b(t)=T^{2} a(t) \in P$, that is, there exist positive numbers $\lambda_{1}, \lambda_{2}$, such that $T a(t) \geqslant \lambda_{1} a(t)$, $T^{2} a(t) \geqslant \lambda_{2} a(t)$. have

Choose a positive number $\lambda_{0} \leqslant\left\{1, \lambda_{1}, \lambda_{2}^{4 / 3}\right\}$ and combining the monotonicity of $T$, we

$$
\begin{gathered}
T\left(\lambda_{0} a(t)\right) \geqslant T a(t) \geqslant \lambda_{1} a(t) \geqslant \lambda_{0} a(t), \\
T^{2}\left(\lambda_{0} a(t)\right) \geqslant \lambda_{0}^{1 / 4} T^{2} a(t) \geqslant \lambda_{0}^{1 / 4} \lambda_{2} a(t) \geqslant \lambda_{0} a(t) .
\end{gathered}
$$


Taking $m(t)=\lambda_{0} t^{1 / 2}$, then,

$$
\begin{aligned}
n(t)=\operatorname{Tm}(t) & =\int_{0}^{1} G(t, s) \phi_{q}\left(\int_{0}^{1} H(s, \tau) f\left(\tau, \lambda_{0} \tau^{1 / 2}\right) d \tau\right) d s \\
& \geqslant \lambda_{0} t^{1 / 2}=m(t), \\
\operatorname{Tn}(t)=T^{2} m(t) & =\int_{0}^{1} G(t, s) \phi_{q}\left(\int_{0}^{1} H(s, \tau) f\left(\tau, T\left(\lambda_{0} \tau^{1 / 2}\right)\right) d \tau\right) d s \\
& \geqslant \lambda_{0} t^{1 / 2}=m(t),
\end{aligned}
$$

that is to say, the condition $\left(\mathrm{H}_{3}\right)$ holds. Theorem 3.1 implies that the fractional boundary value problem (4.1) has at least one positive solution.

\section{Acknowledgments}

This work was jointly supported by Science Foundation of Hunan Provincial under Grants 2009JT3042, 2010GK3008 and 10JJ6007, the Construct Program of the Key Discipline in Hunan Province, Aid Program for Science and Technology Innovative Research Team in Higher Educational Institutions of Hunan Province.

\section{References}

[1] D. Ma and X. Yang, "Upper and lower solution method for fourth-order four-point boundary value problems," Journal of Computational and Applied Mathematics, vol. 223, no. 2, pp. 543-551, 2009.

[2] S. Chen, W. Ni, and C. Wang, "Positive solution of fourth order ordinary differential equation with four-point boundary conditions," Applied Mathematics Letters, vol. 19, no. 2, pp. 161-168, 2006.

[3] X. Zhang and L. Liu, "Positive solutions of fourth-order four-point boundary value problems with p-Laplacian operator," Journal of Mathematical Analysis and Applications, vol. 336, no. 2, pp. 1414-1423, 2007.

[4] Z. B. Bai and Haishen Lü, "Positive solutions for boundary value problem of nonlinear fractional differential equation," Journal of Mathematical Analysis and Applications, vol. 311, no. 2, pp. 495-505, 2005.

[5] S. Q. Zhang, "Positive solutions for boundary-value problems of nonlinear fractional differential equations," Electronic Journal of Differential Equations, no. 36, pp. 1-12, 2006.

[6] J. Wang, H. J. Xiang, and Z. Liu, "Positive solutions for three-point boundary value problems of nonlinear fractional differential equations with $p$-Laplacian," Far East Journal of Applied Mathematics, vol. 37, no. 1, pp. 33-47, 2009.

[7] M. Benchohra, S. Hamani, and S. K. Ntouyas, "Boundary value problems for differential equations with fractional order and nonlocal conditions," Nonlinear Analysis. Theory, Methods E Applications, vol. 71, no. 7-8, pp. 2391-2396, 2009.

[8] S. Liang and J. Zhang, "Positive solutions for boundary value problems of nonlinear fractional differential equation," Nonlinear Analysis. Theory, Methods E Applications, vol. 71, no. 11, pp. 55455550, 2009.

[9] I. Podlubny, Fractional Differential Equations, vol. 198 of Mathematics in Science and Engineering, Academic Press, San Diego, Calif, USA, 1999.

[10] S. G. Samko, A. A. Kilbas, and O. I. Marichev, Fractional Integrals and Derivatives, Gordon and Breach Science Publishers, Yverdon, Switzerland, 1993. 


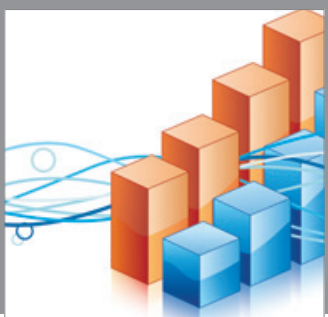

Advances in

Operations Research

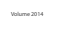

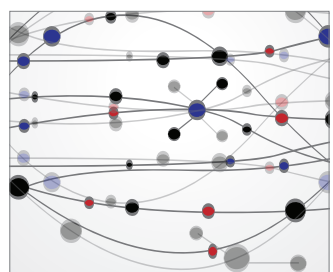

\section{The Scientific} World Journal
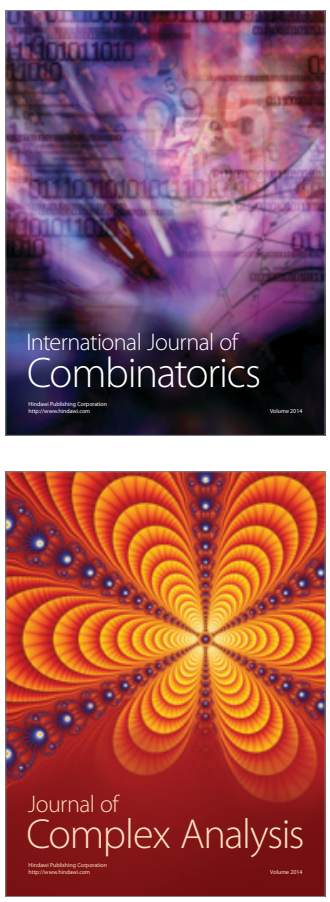

International Journal of

Mathematics and

Mathematical

Sciences
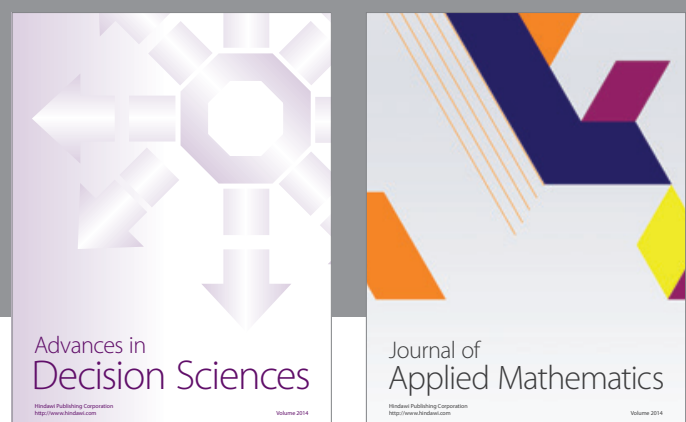

Journal of

Applied Mathematics
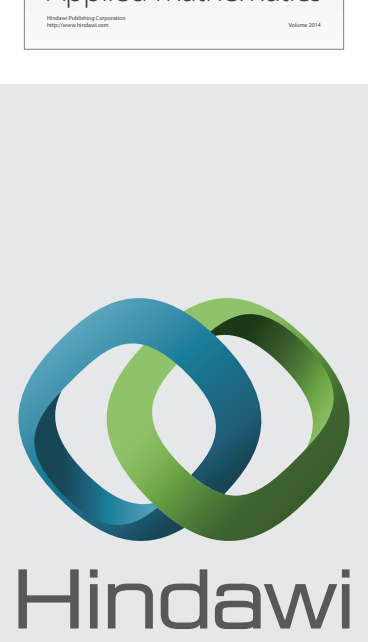

Submit your manuscripts at http://www.hindawi.com
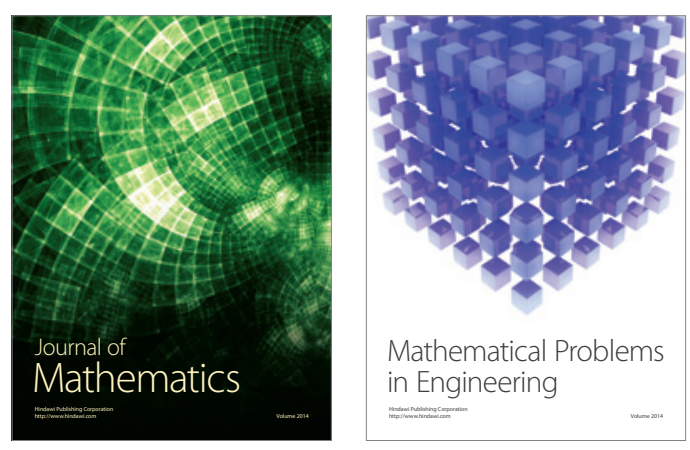

Mathematical Problems in Engineering
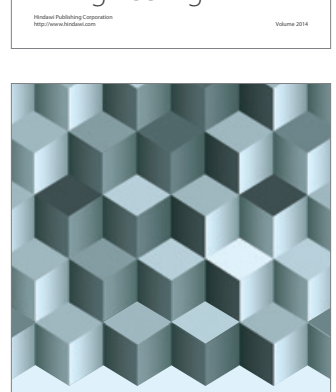

Journal of

Function Spaces
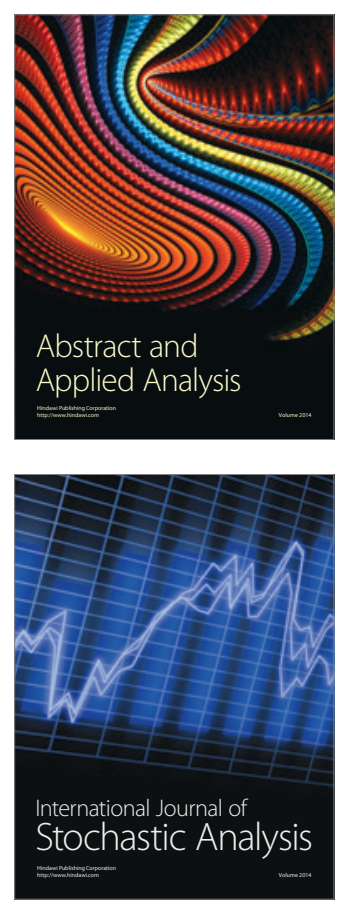

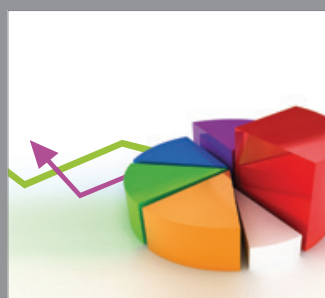

ournal of

Probability and Statistics

Promensencen
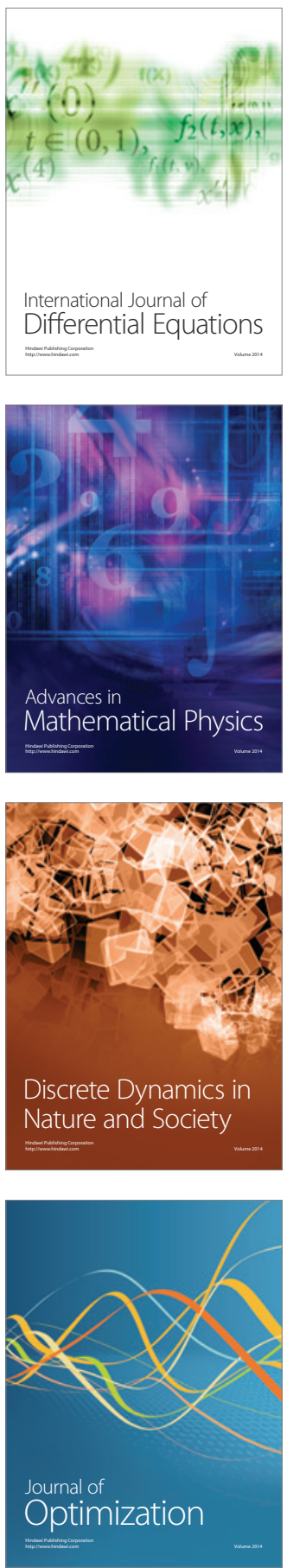